\title{
Les « Roman Jakobson Papers » au Massachusetts Institute of Technology
}

\section{Giuseppe D'Ottavi}

\section{(2) OpenEdition}

1 Journals

Édition électronique

URL : http://journals.openedition.org/genesis/3182

DOI : 10.4000/genesis.3182

ISSN : 2268-1590

Éditeur :

Presses universitaires de Paris Sorbonne (PUPS), Société internationale de génétique artistique littéraire et scientifique (SIGALES)

\section{Édition imprimée}

Date de publication : 15 décembre 2018

ISBN : 979-10-231-06282

ISSN : 1167-5101

\section{Référence électronique}

Giuseppe D'Ottavi, « Les « Roman Jakobson Papers » au Massachusetts Institute of Technology », Genesis [En ligne], 47 | 2018, mis en ligne le 15 janvier 2019, consulté le 21 janvier 2021. URL : http:// journals.openedition.org/genesis/3182 ; DOI : https://doi.org/10.4000/genesis.3182

Ce document a été généré automatiquement le 21 janvier 2021.

Tous droits réservés 


\title{
Les « Roman Jakobson Papers » au Massachusetts Institute of Technology
}

\author{
Giuseppe D'Ottavi
}

1 En 1980, Roman Jakobson (1896-1982), alors professeur émérite du Massachusetts Institute of Technology, remit à la section «Archives et collections spéciales » du MIT un paquet contenant environ 200 lettres et cartes postales que le prince N.S. Troubetzkoy (1890-1938) lui avait envoyées entre 1920 et 1938. Ce matériau, qui avait été à la base de l'ouvrage N.S. Trubetzkoy's Letters and Notes ${ }^{1}$, a été le premier noyau du fonds d'archives «Roman Jakobson Papers ${ }^{2}$ ».

2 Aujourd'hui - après cinq autres donations, dont la dernière en 2012 -, ce fonds nous apparaît comme l'un des plus importants et riches du panorama des archives de linguistes $\mathrm{du} \mathrm{xx}^{\mathrm{e}}$ siècle et, plus généralement, des archives de sciences humaines.

L'arc temporel qu'il couvre est très vaste, plus étendu même que la vie de Jakobson : les documents les plus anciens datent de la fin des années 1880 (ils concernent sa famille), alors que les plus récents (ayant trait aux affaires de la Jakobson Trust) dépassent de peu 1986, année de la mort de sa dernière femme, Krystyna Pomorska (1928-1986). Entre ces deux extrêmes, se déploie un ensemble dont l'importance matérielle (4 mètres cubes environ, comme le détaillent les Archives du MIT) et la variété (manuscrits autographes et imprimés, documents officiels, tapuscrits, ronéotypes, photographies, documents multimédias... en langue russe, tchèque, polonaise, allemande, française, anglaise...) témoignent, en première instance, d'une conscience d'archives très marquée. Ainsi, ce fonds est en mesure d'offrir une vision du cours entier de la vie et de la carrière de Roman Jakobson, une carrière qui se confond avec l'histoire (et la géographie) de la linguistique.

4 Ce véritable trésor n'a pas encore fait l'objet d'une étude critique étendue et ciblée ; il n'a été approché par les chercheurs que de manière occasionnelle. Pourtant, sa valeur peut difficilement être surestimée, non seulement en tant que témoignage de l'œuvre 
de l'un des linguistes du $\mathrm{xx}^{\mathrm{e}}$ siècle les plus renommés, mais aussi, à travers lui, comme véritable source première de l'histoire des sciences humaines tout court.

C'est à Stephen Rudy (1949-2003), professeur de russe et de langues slaves, ami et collaborateur de Jakobson, que l'on doit le premier classement du fonds, alors que le véritable travail d'inventaire - achevé en septembre 1986 - a été l'œuvre d'une équipe d'archivistes du MIT et d'experts de langues de l'Europe de l'Est (Jana Heffernan, Dana Hajdu, Victor Shteynbok, Donna Webber). Le « Guide to the Papers of Roman Jakobson » qui en ressort se signale par le soin extrême et la clarté, ce qui en fait non seulement une excellente porte d'entrée du fonds, mais aussi une véritable étude d'ensemble, la plus complète disponible à ce jour.

6 L'unité archivistique inférieure est la chemise («folder»); à l'intérieur de chaque chemise est rassemblé, sur base thématique, un nombre variable d'imprimés ou de manuscrits (aucun de ces derniers n'est folioté). Exception faite des documents multimédias ou hors format, dans leur grande majorité, les chemises sont réparties dans 137 boîtes cartonnées partagées à leur tour, toujours selon un critère thématique, en 12 sections ("series»). Les sections 1 à 5 rassemblent les documents témoignant de la vie et de la carrière académique de Jakobson et les sections 7, 9 et 10 recueillent des essais (publiés et inédits), tirés à part, comptes rendus et articles de journaux écrits par d'autres et portant sur Jakobson et sur son œuvre. Dans la section 11 se trouve un large ensemble de notes de recherche sous forme d'un nombre indéfini de petites fiches bibliographiques que Jakobson n'a jamais cessé de rédiger et d'utiliser tout au long de sa vie.

7 Les documents ayant trait aux contributions scientifiques de Jakobson ont tous été groupés dans la section 6. Identifié par la référence à la bibliographie institutionnelle ${ }^{3}$, à peu près chaque écrit publié (article de presse, essai, ouvrage...) est entouré des matériaux qui en ont devancé et suivi la parution. Le tout est classé selon l'ordre suivant: premières éditions, réimpressions, éditions ultérieures, traductions; manuscrits et/ou tapuscrits finaux; brouillons, notes de recherches et de travail; sources et matériaux externes; correspondances concernées. Ce principe de rangement "génétique » avant la lettre est dû à Jakobson lui-même qui l'avait conçu et mis en œuvre lors de la préparation des Selected Writings ${ }^{4}$. Parmi les dossiers les plus riches, on compte celui construit autour de La Geste du prince Igor $^{5}$ (dont la documentation comprend jusqu'aux lettres d'envoi d'un exemplaire aux directeurs de diverses bibliothèques, et leurs réponses aussi) et de Preliminaries to Speech Analysis: de cet ouvrage notamment on suit l'évolution complexe, du projet de description analytique de la phonologie du russe contemporain mis en place à Harvard (1950) jusqu'à la première édition parue comme "Technical Report » du Laboratoire d'acoustique du MIT (1952) pour arriver, à travers plusieurs autres étapes, à la dernière édition entièrement revue publiée en $1963^{6}$. De même, chaque phase du travail de conception, élaboration, rédaction et relecture, travail qui s'échelonne sur plus de trente ans, de l'un des sommets de la production jakobsonienne - The Sound Shape of Language ${ }^{7}$-, est entièrement documentée, ici aussi, au-delà même de la date de parution de l'ouvrage. Les inédits trouvent également place dans cette même section 6 : classés par ordre chronologique eux aussi, brouillons, notes de recherche, notes et textes de cours et conférences témoignent des projets inachevés, d'articles et d'ouvrages jamais publiés, même s'ils se trouvent - très souvent - dans un état de rédaction assez avancé. 
8 La section 8 a été constituée en rassemblant la correspondance générique, c'est-à-dire non liée à un projet spécifique : compte tenu de l'étendue temporelle (la dernière lettre date de l'année de la mort de Jakobson) et des noms impliqués (Leonard Bloomfield et Umberto Eco, Claude Lévi-Strauss et Vladimir Nabokov, par exemples), cet ensemble représente, à lui seul, une source incontournable pour l'histoire des sciences humaines.

Finalement, dans la section 12, sont rassemblés les matériaux venant de la dernière acquisition. Ils sont inventoriés de manière encore sommaire: on y trouve, entre autres, une partie des documents officiels témoignant de l'affaire de soupçon d'espionnage dans laquelle Jakobson a été impliqué par le FBI au cours des années cinquante.

Jakobson a également légué au MIT sa bibliothèque personnelle. Actuellement, seule une partie minime est cataloguée en tant que telle: les outils de consultation des bibliothèques du MIT ne donnent les détails que de quatre-vingts ouvrages classés comme ayant appartenu à Jakobson, alors qu'une recherche empirique a pu localiser de nombreux autres volumes portant son ex-libris (parmi eux, les œuvres d'Émile Benveniste, Otto Jespersen, Daniel Jones, Louis Hjelmslev et autres, avec dédicaces et/ ou signes de lecture). Un ouvrage se détache de tout le reste : il s'agit d'un exemplaire du Cours de linguistique générale de Ferdinand de Saussure $\left(1922^{2}\left[1916^{1}\right]\right)$, avec note de possession autographe de Jakobson ${ }^{8}$. Ayant évidemment suivi Jakobson de Moscou à Prague ${ }^{9}$ et aux États-Unis, cet exemplaire du Cours devient le support de la diffusion du structuralisme dans le monde, et cela pas seulement symboliquement. Il est rare qu'on puisse relier le destin d'un courant intellectuel à un seul objet matériel, mais tel est le cas de l'exemplaire du Cours ayant appartenu à Jakobson et aujourd'hui conservé dans les magasins du MIT.

11 À ce jour, les archives Jakobson au MIT ne sont que faiblement exploitées. Au-delà de quelques notices génériques ou mentions de passage ${ }^{10}$, seule la correspondance a fait l'objet de publications spécifiques ${ }^{11}$, alors qu'un article isolé fait valoir l'intérêt historique et les potentialités génétiques du fonds ${ }^{12}$. Au printemps 2017, une mission de recherche financée par le LabEx TransferS dans le cadre du projet "Genèse et transferts de savoirs linguistiques" a permis à l'équipe "Linguistique » de l'ITEM d'entreprendre une première exploration des «Roman Jakobson Papers» dans le but d'ouvrir la voie à leur étude systématique. Parmi les premiers projets particuliers mis en chantier, l'étude des notes entourant le travail sur « Les Chats » de Baudelaire (mené avec Claude Lévi-Strauss) ${ }^{13}$, l'édition intégrale de la correspondance avec la phonologue danoise Eli Fischer-Jørgensen (1911-2010), la publication des conférences inédites de Tokyo $(1967)^{14}$ et de la transcription (revue par lui-même) d'un long entretien diffusé à la télévision française entre 1972 et $1974^{15}$, où Jakobson fait preuve de son talent de metteur en scène d'auto-narration, genre dans lequel il excellait. 


\section{NOTES}

1. The Hague / Paris, Mouton, 1975 (éd. fr. par Patrick Sériot : N.S. Trubeckoj, Correspondance avec Roman Jakobson et autres écrits, Lausanne / Paris, Payot, 2006).

2. MIT Archives and Special Collections, MC.0072.

3. Stephen Rudy, Roman Jakobson 1896-1982. A Complete Bibliography of his Writings, Berlin / New York, Mouton / de Gruyter, 1990.

4. 8 vol. , La Haye / Paris / New York / Berlin / Amsterdam / New York, Mouton / de Gruyter, 1962-1988.

5. Texte établi, traduit et commenté sous la direction d'Henri Grégoire, Roman Jakobson et Marc Szeftel, assisté de J. A. Joffe, New York, Columbia University Press, 1948.

6. Avec C. Gunnar M. Fant et Morris Halle, Cambridge, MIT Press, 1963 [1952 $\left.{ }^{1}, 1955^{2}, 1961^{3}\right]$.

7. Avec Linda R. Waugh, Bloomington, Indiana University Press / Hassocks, Harvester Press, 1979.

8. Institute Archives - Non circulating Collection 1, PS121.S259 1922.

9. Voir dernièrement Alessandro Chidichimo et Estanislao Sofía, «À propos des traductions, la diffusion et la réception du Cours de linguistique générale en Russie (1916-1927) », dans V. Chepiga et E. Sofía (dir.), La Correspondance entre linguistes. Un espace de travail, Louvain-la-Neuve, Academia-L'Harmattan, 2017, p. 155-178.

10. Voir H. Baran, S. Gindin, N. Grinzer, T. Nikolaeva, S. Rudy et E. Shumilova (dir.), Roman Jakobson. Teksty, dokumenty, issledovaniya [Roman Jakobson. Texts, Documents, Studies], Moscou, Rossiiskii Gosudarstvennyi Gumanitarnyi Universitet [Université d'État Russe pour les Sciences Humaines], 1999 ; M. Thomas, «Jakobson's Circles », dans V. Kasevich, Y. A. Kleiner et P. Sériot (dir.), History of Linguistics 2011 : Selected Papers from the $12^{\text {th }}$ International Conference on the History of the Language Sciences (ICHoLS XII), Saint Petersburg, 28 August-2 September 2011, Amsterdam, Benjamins, 2014, p. 145-155.

11. Voir Morris Halle, "The Bloomfield-Jakobson Correspondence, 1944-1946 ", Language 64/4 (1988), p. 737-754 ; J. Toman (dir.), Letters and Other Materials from the Moscow and Prague Linguistic Circles, 1912-1945, Ann Arbor, Michigan Slavic Publications, 1994 ; Henryk Baran, « Vokrug "Slovo o polku Igoreve" : Iz perepiski R.O. Jakobsona i A.V. Solov'eva » [Autour de La Geste du prince Igor : de la correspondance entre Roman Jakobson et Alexander Soloviev], Slavianovedenie 4, 2000, p. 50-78; Cristina Altman, «A correspondência Jakobson-Mattoso Câmara (1945-1968)", Confluência 49 (2015), p. 9-42 ; Roman Jakobson, Claude Lévi-Strauss, Correspondance. 1942-1982, éd. par E. Loyer et P. Maniglier, Paris, Le Seuil, 2018.

12. John E. Joseph, «The Genesis of Jakobson's Six Leçons », Historiographia Linguistica 16/3 (1989), p. $415-420$.

13. L'Homme 2 (1962), p. 5-21.

14. Il s'agit de dix conférences données dans le cadre du II International Seminar in Linguistic Theory, plus un discours d'ouverture.

15. Archives du $\mathrm{XX}^{e}$ siècle, par Jean-José Marchand. 
INDEX

Mots-clés : Roman Jakobson, Archives, Manuscrits, Structuralisme, Linguistique

\section{AUTEUR}

\section{GIUSEPPE D'OTTAVI}

GIUSEPPE D’OTTAVI, membre associé de l'équipe « Linguistique » de l'ITEM, est actuellement chercheur à l'université de Copenhague où il travaille à l'édition de la correspondance de Roman Jakobson. Il codirige avec Irène Fenoglio (ITEM) la collection « Sciences du langage. Carrefours et points de vue » chez Academia-L'Harmattan (Louvain-la-Neuve). Parmi ses dernières publications : «Matériaux pour l'étude de De l'emploi du génitif absolu en Sanscrit de Ferdinand de Saussure (1881) » (Cahiers Ferdinand de Saussure 69 [2016]) ; «Pour une théorie benvenistienne de l'écriture : enquête philologico-historique ", dans G. D'Ottavi et I. Fenoglio (dir.), Émile Benveniste, 50 ans après les Problèmes de linguistique générale (Éditions Rue d'Ulm, Paris, 2018) ; « Benveniste entre les lignes. Contribution à l'étude de l'imaginaire linguistique des linguistes » (CMLF 2018) avec R. Hérout ; Le Cours de linguistique. Formes, genèses et interprétations de notes d'auditeurs (Langages 209 [2018]), éd. avec P.-Y. Testenoire.

giuseppe.dottavi@ens.fr 